\title{
European Polymer Journal announces new section on macromolecular nanotechnology
}

The aim of European Polymer Journal (EPJ) has been to act as a platform for the exchange of research results in macromolecular science and technology defined in a very broad sense. Whereas at present the publisher and the editors of EPJ are committed to maintain the breadth of the journal's scope, we wish to launch a separate section of the Journal focusing on Macromolecular Nanotechnology and bundle related articles in it dedicated to this topic. This section will encompass original papers (communications and full articles) focusing on fundamental research, nanoscale technologies, nanostructured polymeric materials, and biological nanosystems, as well as review articles (usually upon invitation by the editors) reflecting major achievements and trends in macromolecular science and technology at the nanoscale. It appears to us that certain areas in polymer science and technology have become mature and new frontiers for polymers are emerging in nanoscience, nanotechnology, and bio-macromolecular science and engineering. For example, the number of articles dealing with nanostructured polymer materials such as nanocomposites, published in $E P J$, is growing at an unprecedented rate. While some of these submissions revisit old problems under new names using a "nano" nomenclature, there are also many truly original manuscripts which focus on the cutting edge of nanostructured polymers. We believe that the related paradigm shifts must be accompanied by a changing emphasis of our journal profile. With this new section we respond to the growing needs of the scientific community to have a dedicated platform in EPJ to publish results in nanoscience and nanotechnology of macromolecular substances in a dedicated Journal section.

There is more than one way to define nanoscience and nanotechnology. First the length scale needs to be specified. Somewhat arbitrarily, the definition of systems with at least one dimension in approximately the 1-100 nanometer range is usually considered as nanoscale. The overall objective of nanoscience is to seek fundamental scientific understanding of phenomena, materials, and relationships, which govern the behavior of matter at this length scale. Nanotechnology aims at the fabrication, characterization and use of structures, devices and systems that have novel properties and functions because of their miniature size. For bottomup nanotechnologies it is essential to have a direct control of matter either between two nanoobjects, or between a micro (or macro) object and a nanoobject. Novel scanning probe technologies, such as Atomic Force Microscopy (AFM), as an enabling method, allow one to measure forces that act on a single macromolecule, or a section of it, while being stretched. AFM is also an enabling platform for nanofabrication using various lithography approaches to manipulate molecules with nanometer precision. Single molecule optical techniques are emerging as nanoscale probes of soft materials and allow one to study macromolecules from a true chain segment perspective, as opposed to conventional ensembleaveraging molecular techniques, like scattering. Often the building elements in bottom-up nanotechnology are single macromolecules like dendrimers, which can now be manipulated at the molecular scale with atomic precision using the aforementioned fabrication platforms. All these, and other related topics will be covered in EPJ's new section.

Polymeric materials often exhibit a structural hierarchy with distinct features having dimensions at the nanoscale. Furthermore, fillers, additives, reinforcing agents, etc. with nanoscale dimensions are often added to polymers to change their properties in a desired way. These materials are "per definitio" nanostructured and have been around for a long 
time, i.e. they do not really represent a new class of matter. However, due to a rapid development of controlled and predictive manufacturing of nanosize additives and directed fabrication at the nanoscale, true nanoscale engineering of polymeric materials has only recently come within our grasp.

In EPJ's Macromolecular Nanotechnology section we shall also promote articles dealing with bio-macromolecules. The goals of bio-nanotechnology with macromolecules include enhancing our understanding of the structure and function of biological polymer molecules, and to adopt nature's solutions in biomimicking macromolecular systems to advance technology. Applications may include molecular motors, new devices, bioelectronics, arrays for biomacromolecular sensing, polymerbased nanoscale release systems, nanostructured polymeric biomaterials for implant and tissue engineering applications, etc.
EPJ's Macromolecular Nanotechnology section will include articles in the areas outlined above i.e. papers that fit into our broad definition as sketched above. We plan to launch the first EPJ section on Macromolecular Nanotechnology around the middle of 2006 and hope that publishing articles at this forefront of macromolecular nanoscience and nanotechnology will further strengthen the current trend of massive growth of our Journal's impact factor.

Editor

G. Julius Vancso

University of Twente, Department of Materials Science and Technology of Polymers and MESA+ Institute for Nanotechnology, P.O. Box 217, 7500 Enschede, Netherlands. Tel.: +31 53489 2974; fax: +31 534893823.

E-mail address: g.j.vancso@tnw.utwente.nl
The launch of the new section "Macromolecular Nanotechnology" represents a landmark in the life of European Polymer Journal. This emerging area is becoming an increasingly important part of polymer science. European Polymer Journal has had a successful 2005, once again increasing its impact factor (from 1.086 to 1.419) as well as making advances in its scope of topics and range of authors. We welcome the new editors Professors Gedde and Clarson who both add considerable gravitas to EPJ. We would like to thank all of our authors who have supported the journal during 2005 and look forward to receiving your manuscripts in 2006. We would also like to thank Rumen Duhlev and all of his associates at Elsevier who continue to provide excellent support for EPJ.

Editor-in-Chief

David M. Haddleton

Department of Chemistry, University of Warwick, Coventry CV4 7AL, United Kingdom. Tel.: +44 2476 523256; fax: +442476 528267. E-mail address: d.m.haddleton@warwick.ac.uk 